\title{
Automated Development of Individual Learning Paths on the Competency Approach
}

\author{
http://dx.doi.org/10.3991/ijep.v3i4.2900 \\ A.E. Shukhman, M.V. Motyleva and I.D. Belonovskaya \\ Orenburg State University, Orenburg, Russia
}

\begin{abstract}
This article proposes new approach to the development of student's individual learning path (ILP). The result of student's learning is defined as a set of competencies levels. The use of the multi-level system of generalized professional competencies allows to model ILP effectively and to automate the development of an optimal ILP. Each competency level in our model introduces some substantial units called subunits. The process of learning is a sequence of transitions from one student's state to another through a study of one or more courses. It is possible to present the relation of competency levels and courses in our model as Petri net. Using the Petri net model it is possible to automatically generate the individual learning path including the courses that the student needs to attend according to the set of certain competency levels. For searching of an optimum individual learning path we use algorithm based on tree of reachability for Petri net. The developed model and algorithm were used in the prototype of the information system for automating the construction of individual learning paths. The developed information system may be used for the construction of individual student's learning paths in educational institutions of higher education.
\end{abstract}

Index Terms-individual learning path, system of competencies, Petri nets.

\section{INTRODUCTION}

Now university educational programs include mandatory study courses and variable choice courses. Each student creates his or her individual learning path by choosing some courses at the beginning of the academic year or semester. Let's define the student's individual learning paths (ILP) as the set of courses that form a partially ordered sequence.

Often students have trouble selecting courses. They do not consider the contribution of certain courses in their future qualification and thus violate logical relations of courses. Student's learning path may be adjusted during training.

The result of student's learning is defined as a set of competency levels. Professional competency is the clearly formulated set of professional practical skills that a student is supposed to have at graduation. The use of the multi-level system of generalized professional competencies allows to model ILP effectively and to automate the development of an optimal ILP.

The common professional standards are not to be used for the development of educational content because knowledge and skills only but not competencies are considered as qualification characteristics. Besides being too many knowledge and skills do not form a system. Common competencies for a number of professions are not identified. The development of competencies system is based on educational standards, professional standards, employer requirements and effective professional educational programs.

We have based our system structure of generalized professional competencies on the results of professional staff activity: a material product, an information (software) product, a psychological product, a financial product, an innovative idea, an innovative technology, an innovative project, an innovative process, an innovative method, an innovative tool, an arrangement of one's personal work, personnel management. For each result of professional activity we have determined generalized skills representing key subunits of competency levels [1].

Comparing these results with levels of education we have been able to identify five levels of competency included in each other in process of complexification: readiness for practical typical actions on a pattern; readiness for the regulated independent actions; readiness for applied independent practical activities in innovative conditions; readiness for design, development, and realization of innovative products; readiness for research and forecasting in the sphere of innovations.

Substantial units (knowledge, skills) are introduced in our model to establish formal compliance between courses of the curriculum and professional competencies. Each substantial unit is included into the content of competency at a certain level and also into the content of one or several courses of the curriculum. Competencies may determine extended requirements that are needed for the learning several interdependent courses.

The subject domain analysis let to develop an information system for storage and processing of generalized competencies, providing modeling competency systems as well as the curriculums. One of the features of our system is the development of students' optimal ILP.

\section{ForMALIZATION OF THE PROBLEM OF CONSTRUCTING INDIVIDUAL LEARNING PATHS}

The result of education on a certain specialization is described in our model as a set of certain competency levels. It can be formed from multiple sets of:

1) mandatory competencies for all students;

2) competencies of additional qualification;

3 ) additional competencies formed in choice courses. 
A student choice is based on the employer requirements and his or her own aptitudes and preferences.

A set of initial levels of professional competencies must be determined for students. Initial level of competencies is different for students receiving a first professional training and those already have a certain level of professional education.

The content of competencies should be formalized for building a mathematical model. The requirements for training results are defined as a set $C$ of levels of competencies $c_{j}$ a graduate should have:

$$
C=\bigcup_{j=1}^{n} c_{j} .
$$

Any competency at certain level can be represented as a set of one or more subunits. A subunit is an independent part of one competency representing one specific requirement for the result of education (knowledge, skills). Let $e_{j i}$ be a substantial unit (subunit) of competency $c_{i}$ then

$$
c_{i}=\bigcup_{j} e_{i j} .
$$

Let's define the set $S$ as the set of objects of study (for example programming languages, databases, computer networks, etc.) Consider a set of functions $T=\{\tau\}$, where each function $\tau: S \rightarrow E$ identifies specific actions on objects of study (for example to know, to use, to develop, etc.) As an example, the object of study can be "databases" and the function can be "to use".

A function $\tau: S \rightarrow E$ is isomorphic under the operation of the set union:

$$
\tau\left(s_{1} \cup s_{2}\right)=\tau\left(s_{1}\right) \cup \tau\left(s_{2}\right) .
$$

Subunit of competency is determined as a result of applying the function to the object of study

$$
e_{j i}=\tau\left(s_{j i}\right), e_{j i} \in E, s_{j i} \in S, \tau \in T .
$$

Through (3) we rewrite (1) in the form

$$
c_{j}=\bigcup_{i} \tau_{i}\left(s_{j i}\right)
$$

Objects of study for all subunits of one competency level are different:

$$
\begin{aligned}
& c_{j}=\bigcup_{i} e_{j i}, \forall e_{j i} \in c_{j}, e_{j i}=\tau_{i}\left(s_{j i}\right), \\
& \forall e_{j l} \in c_{j}, e_{j l}=\tau_{i}\left(s_{j l}\right), s_{j i} \neq s_{j l}, i \neq l .
\end{aligned}
$$

The content of academic course should be modeled in accordance with the competency approach.

Let $D=\left\{d_{i}\right\}$ is set of courses for construction ILP, $p \in d_{i}$ is a topic within the course $d_{i}, \mu: S \rightarrow D$ is expert-determined function that assigns a object of study $s_{k}$ to set of topics $P \subset D$

$$
\begin{aligned}
& d_{i}=\bigcup_{j} p_{i j}, D=\bigcup_{i} d_{i}=\bigcup_{i} \bigcup_{j} p_{i j} ; \\
& \mu\left(s_{k}\right)=P_{k}, P_{k} \subset D, s_{k} \in S .
\end{aligned}
$$

Next we consider a set of competencies $E$ that set of topics for any objects of study either coincide or are disjoint.

$$
\left\{\begin{array}{l}
E=\bigcup_{k=1}^{n} \tau_{k}\left(\bigcup_{i=1}^{i_{k}} s_{j i}\right)=\bigcup_{k=1}^{n} \bigcup_{i=1}^{i_{k}} \tau_{k}\left(s_{j i}\right) \\
\mu\left(s_{j i}\right)=P_{j i}, P_{j i} \subset D, s_{j i} \in S \\
\mu\left(s_{l t}\right)=P_{l t}, P_{l t} \subset D, s_{l t} \in S \\
{\left[\begin{array}{l}
P_{j i}=P_{l t} \\
P_{j i} \cap P_{l t}=\varnothing
\end{array}\right.}
\end{array}\right.
$$

The process of training is presented as a consecutive transition from initial level of competencies to the chosen result through studying academic courses. Studying each course needs the possession of an entrance set of competency levels. That set can be empty. The subunits included into the course content allow students to raise their level of some competencies. Thus each course $d_{i} \in D$ is related to the two sets of subunits:

1) the set of subunits $E_{i}$ a student needs to start learning a specific course (we call it the initial set of requirements);

2) the set of subunits $E_{i}^{\prime}$ a student acquires mastering the course (we call it the final set of requirements).

These two subsets of set $E$ include all subunits that are nonoverlapping for each course. For some basic courses the initial set of requirements can be empty. They can be studied without any competency.

Let's define a student's state in the learning process at $j$-th stage $o_{j}$ as a set of subunits, that he or she at some time:

$$
o_{j}=\left\{e_{j i}\right\}
$$

The process of learning within the competence approach is a sequence of transitions from one student's state to another. Each of these transitions is a study of one or more courses.

The study of a specific course implies the student transition from state $o_{i}$ to state $o_{j}$. Learning process is a sequence of courses from ILP which allows a student to transit from the initial state $o_{0}$ to the final state $o_{m}$.

\section{THE MODEL OF INDIVIDUAL LEARNING PATH DEVELOPING WITH THE USE OF PETRI NETS}

It is possible to present the relation of subunits and courses in our model as a Petri net. Several examples can be found in the papers when Petri nets have been used in the modeling of learning systems $[3,4]$. For example Petri nets are used in order to optimize learning paths to reach a degree or a qualification [5]. However, the authors don't use the competency approach.

In our model the set of competency subunits $E$ determines the set of places in Petri net. The set of courses $D$ determines the set of transitions in Petri net (Fig. 1). 


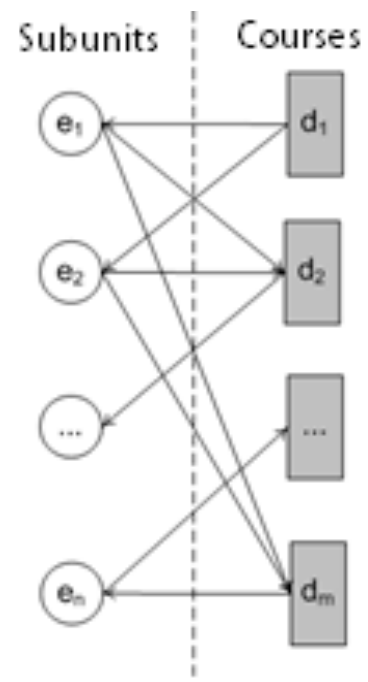

Figure 1. The Petri net for learning process.

The marking of Petri net is a function which associates each place with a label 0 or 1 .

$$
\Phi: E \rightarrow\{0,1\}
$$

A set of student's competency levels corresponds to a certain marking of the Petri net. In our model label value equal to 1 means the presence of a competency subunit, the value of 0 is the absence of a subunit. In our case the Petri net marking determines the student's state at a certain stage of learning. The net marking may change during transition operations.

If each input place of transition $d_{j}$ contains a 1 , the transition $d_{j}$ may fire. When firing the transition all of its output places are marked 1. The process of training is describes by a sequence of transition operations in Petri net.

The arcs in our Petri net can be of four types (Fig. 2):

1) course for the development of several subunits (Fig. 2a);

2) several choice courses to develop of a subunit (Fig. $2 b)$

3) for the beginning of the course it may be necessary to have multiple subunits at the same time(Fig. 2c);

4) one subunit allows the student to study several courses (Fig. 2d).

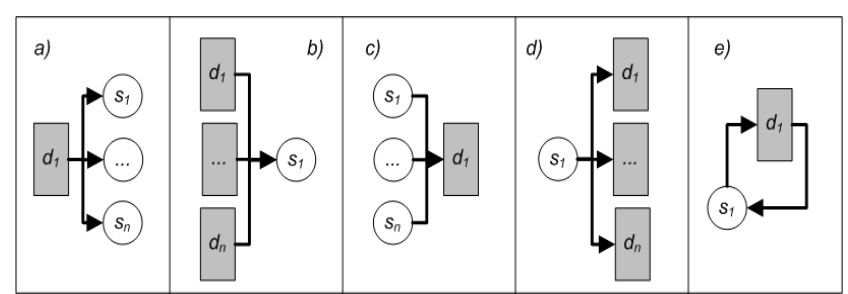

Figure 2. The four types of arcs in our Petri net.

A competency subunit may be formed on the basis of several courses at the same time. For example, in the case shown in Fig. 3, the subunit is formed by studying set of courses $D_{i}^{1}$ or set $D_{i}^{2}$. In this case it is necessary to modify the net by adding imaginary places and transitions.

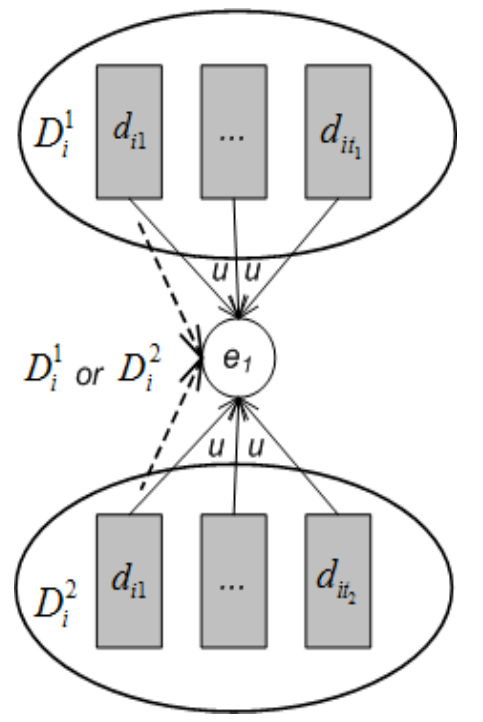

Figure 3. The subunit is formed by several courses

We define $D_{i}=\left\{D_{i}^{(j)} \mid j=1, \ldots, t\right\}$ as the aggregate of sets which are variants for forming subunit $e_{i}$. Let $t=\left|D_{i}\right|$ be quantity of variants and $t_{j}=\left|D_{i}^{(j)}\right|$ is quantity of courses in each set.

First we add the new imaginary subunits as the places:

$$
E_{i j}^{\prime}=\left\{e_{i j}^{(l)} \mid l=1, \ldots, t_{s}\right\}
$$

The quantity of imaginary subunits for each of variants is equal to the quantity of courses in the set.

Next we add the new imaginary courses as the transitions

$$
D_{i}^{\prime}=\left\{d_{i j} \mid j=1, \ldots, t\right\}
$$

Their quantity corresponds to the quantity of variants. Arcs from real courses to the subunit $e_{i}$ pass through the imaginary subunits and courses: $D_{i} \rightarrow E_{i j}^{\prime} \rightarrow D_{i}^{\prime} \rightarrow e_{i j}$ (Fig. 4).

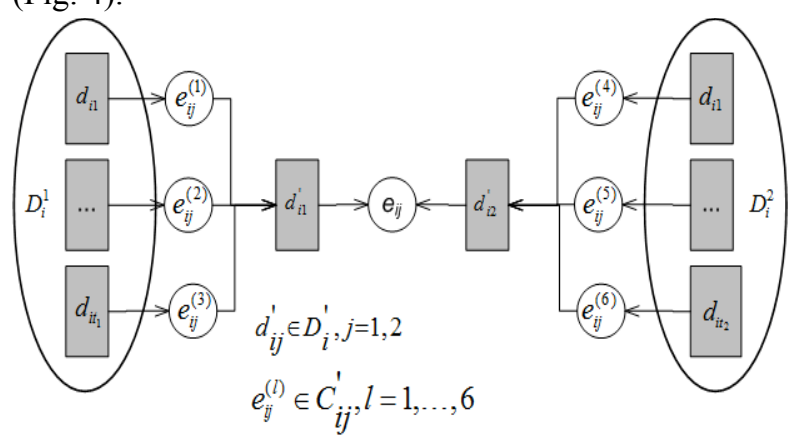

Figure 4. Forming subunit from several courses simultaneously

Thus the problem of constructing individual learning paths is reduced to the construction of transition sequence in the Petri net from the initial state $s_{0}$ to the final state $s_{m}$ (Fig. 5)

Using the Petri net model it is possible to automatically generate the individual learning path including the courses 
that the student needs to attend accordingly to the set of certain competencies levels.

The building a sequence of transitions can be performed using the algorithm for constructing the tree of reachability. This algorithm builds an oriented rooted tree with nodes that will represent all possible markings and the arcs correspond to transitions (courses).

Initial marking corresponds to initial student's competence $o_{0}$. The tree of reachability must be build up to the level where we get a vertex corresponding the final student's state $o_{m}$. Using a breadth-first search for the traverse the tree of reachability gives individual learning paths with the least number of courses. The quantity of Petri nets markings increases exponentially, so if there is a large number of competencies subunits, then it's better to use approximation algorithms based on backtracking to find ways.

For recovery of ILP based on the tree of reachability the courses must be selected on the way from the root node to the final node.

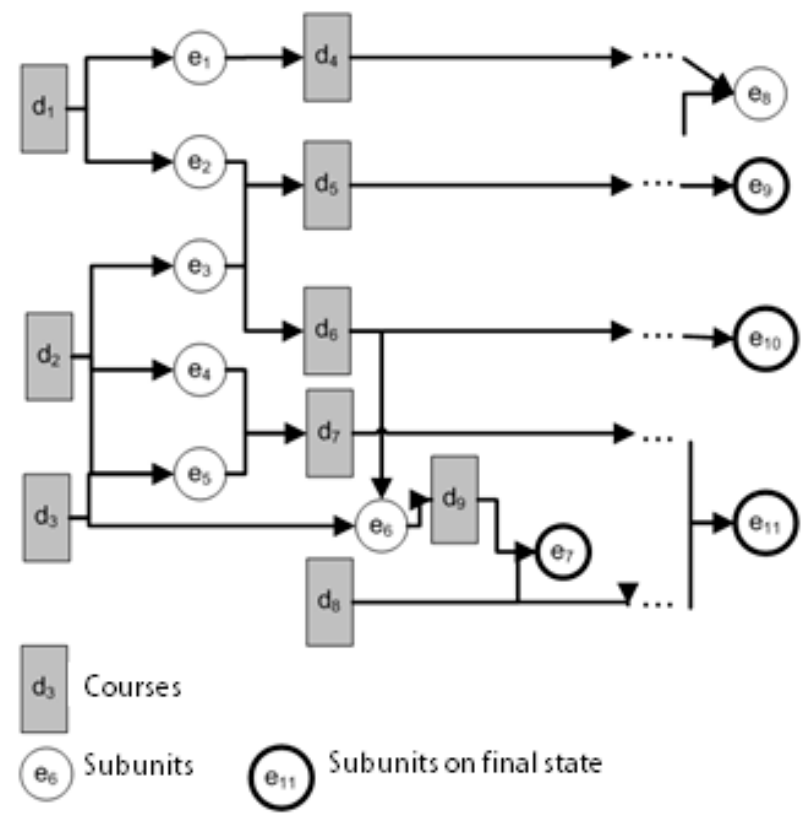

Figure 5. The example of Petri net for ILP

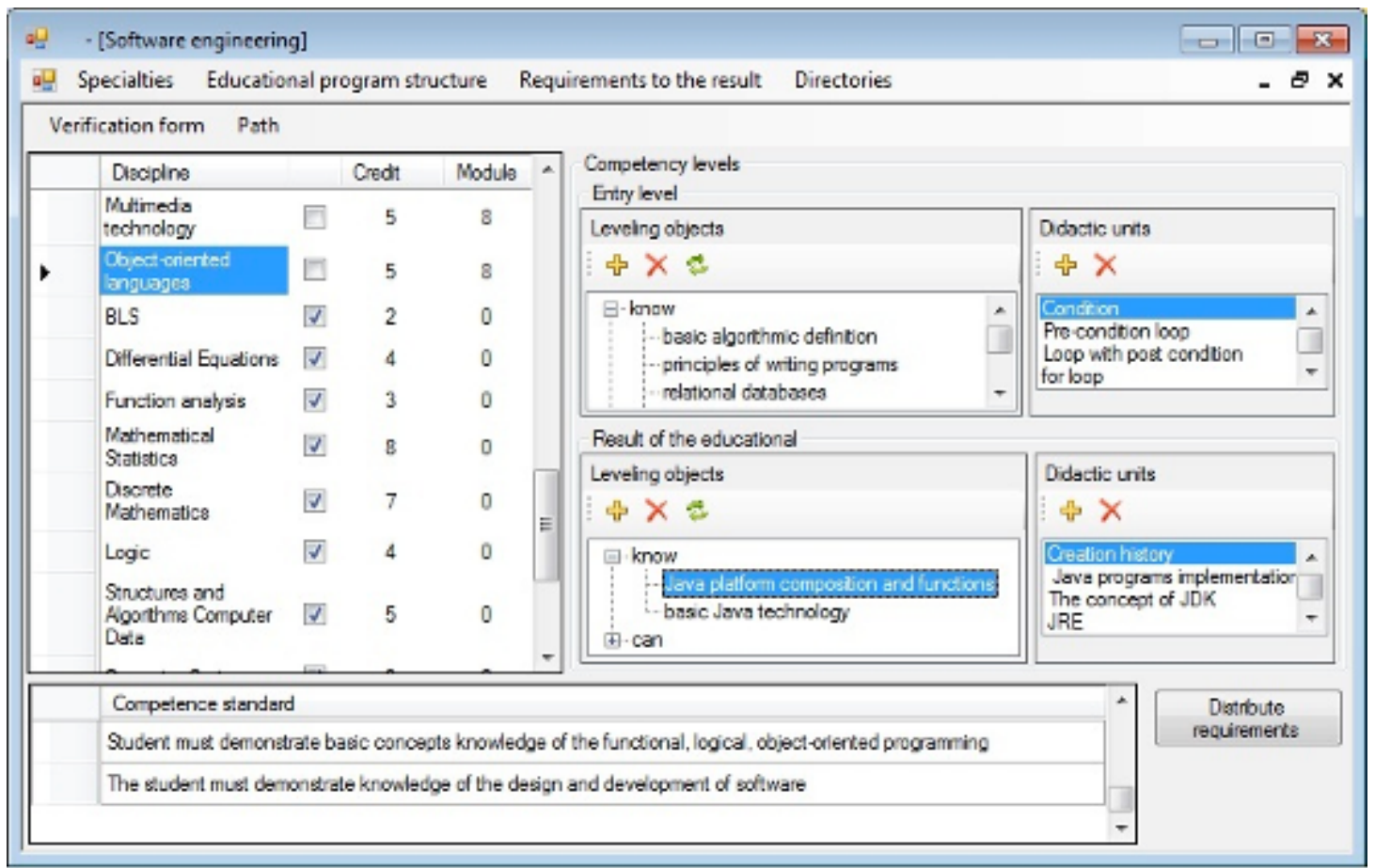

Figure 6. The form for editing the content of educational program. 
SPECIAL FOCUS PAPER

Automated DeVElopment of INDIVIDUAL LEARNING PATHS ON THE COMPETENCY APPROACH

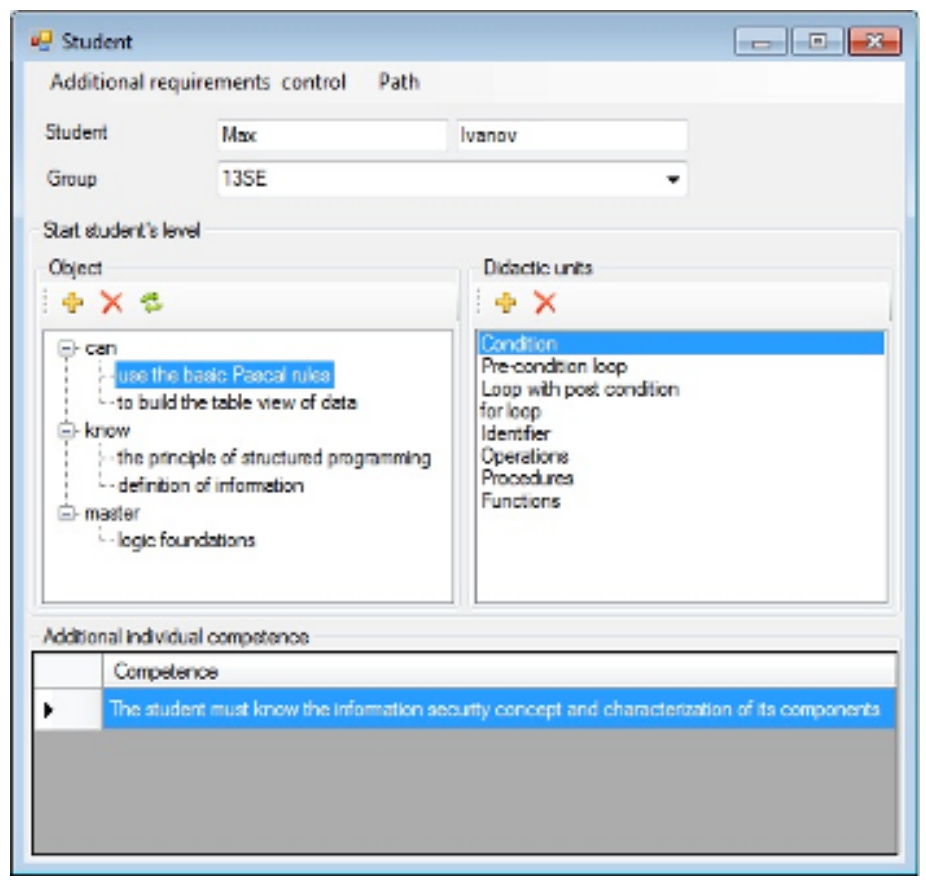

Figure 7. The form for representing the student's individual learning path.

\section{CONCLUSION}

Based on the proposed model, we have developed a prototype of the information system for automating the construction of individual learning paths.

The information model of system has been developed as set of classes. Realization of this information system is done on the basis of the layer conception: layer of business logic, layer of representation, and data storage layer. The user interface of system has been designed and realized.

The information system allows to store and process the educational content in the form of competencies and its subunits, the curriculum as set of courses and topics (Fig.6).

Also this system allows to determine the learning goal for each student and automatically construct an individual learning path (Fig.7)

Currently the prototype of this information system is being tested at the bachelor degree programs "Information Technology" and "Applied Mathematics".

The developed information system may be used for the construction of individual student's learning paths in educational institutions of higher education.

\section{REFERENCES}

[1] A.E. Shukhman., I.D. Belonovskaya, K.E. Tsvetkova "Model of continuous multilevel preparation of specialists for innovation branches of economy", Vestnik Orenburgskogo Gosudastvennogo universiteta, vol. 121, February 2011, pp. 390395

[2] A.E. Shukhman., I.D. Belonovskaya, "Continuous educational programs constructing for training specialists in innovative branches of economy on the basis of generalized competences system", in proceedings of the International Conference on Interactive Collaborative Learning (ICL), IEEE Catalog Number: CFP1223R-USB, ISBN:978-1-4673-2426-7, 26 - 28 Sep. 2012, Villach, Austria.

[3] D.C. Borges, H.B. Neto, J.N de Souza. "Work in progress - Petri Nets as applied to the modeling of E-learning cooperative systems", IEEE Frontiers in Education Conference (FIE), October 27-30, 2010, pp. F1D-1-F1D-3

[4] Z. Baohua "Research on Evaluation of E-Learning Modelling Based on Petri Nets", International Conference on Advanced Computer Theory and Engineering, ICACTE 2008, December 2022, pp. 699-703

[5] R Campos-Rebelo, A Costa, L Gomes "Finding learning paths using Petri nets modeling applicable to E-learning platforms", Technological Innovation for Value Creation, pp. 151-160

\section{AUTHORS}

A. E. Shukhman is head of Department of IT Management, Orenburg State University, Orenburg, Russia (e-mail: ais@mail.osu.ru).

M. V. Motyleva is teacher of Department of IT Management, Orenburg State University, Orenburg, Russia (e-mail: ais@mail.osu.ru).

I. D. Belonovskaya is head of Department for branches and colleges, Orenburg State University, Orenburg, Russia (e-mail: orfk@mail.osu.ru).

This work was supported by the Ministry of Education and Science of Russian Federation (projects 16.740.11.0111 and 14.740.11.0570 by the federal target program "Scientific and scientific-pedagogical personnel of innovative Russia") This article is an extended and modified version of a paper presented at the EDUCON2013 conference held at Technische Universität Berlin, Berlin, Germany from March 13-15, 2013. Submitted 09 June 2013. Published as re-submitted by the authors 11 October 2013. 